\title{
OPTICALLY DETECTED MAGNETIC RESONANCE OF EXCESS ELECTRONS IN CdTe/(Cd,Mg)Te QUANTUM WELLS
}

\author{
C.Y. Hu, W. Ossau, D.R. Yakovlev, B. König \\ Physikalisches Institut der Universität Würzburg \\ Am Hubland, 97074 Würzburg, Germany \\ T. Wojtowicz, G. Karczewsin and J. KossuT \\ Institute of Physics, Polish Academy of Sciences \\ Al. Lotników 32/46, 02-668 Warsaw, Poland
}

\begin{abstract}
The spin resonance of excess electrons is observed with the detection either on the neutral or the negatively charged exciton $X^{-}$emission in type I $\mathrm{CdTe} /(\mathrm{Cd}, \mathrm{Mg}) \mathrm{Te}$ quantum wells with excess electrons of low density. It is found that the electron spin-dependent and electron spin-conserving formation and recombination of $X^{-}$make the optical detection of the spin resonance of excess electrons feasible. For the first time, optically detected magnetic resonance is used to study fast optical transition processes in the nanosecond timescale where the microwave-induced magnetic transition rate is much lower than the optical transition rate.
\end{abstract}

PACS numbers: $76.70 . \mathrm{Hb}, 71.35 .-\mathrm{y}, 71.35 . \mathrm{Ji}$

Optically detected magnetic resonance (ODMR) has been proved to be a useful tool to study recombination processes in bulk semiconductors [1]. The magnetic resonance is detected by monitoring microwave-induced changes in intensity of the luminescence between magnetic sublevels. It is well known that a prerequisite for ODMR is that the rate of the microwave-induced transition between magnetic sublevels has to be higher than or comparable to the optical transition rate. To satisfy this condition, the optical lifetime should be in the sub-microsecond timescale for usual microwave powers available for ODMR measurements. Because of this requirement, ODMR was up to now limited to study relatively slow optical transitions in the microsecond timescale only. Typical examples of such studies are investigations of indirect excitonic transitions in bulk semiconductors [1] or quantum wells (QWs) [2-4]. In this paper we demonstrate that ODMR can be applied to a study of fast optical transitions occurring on the nanosecond timescale, such as direct excitonic transitions in type I QWs containing electron gas of low density. Namely, the excess-electron spin resonance (EESR) is detected on the neutral or 
negatively charged exciton emission with the microwave-induced transition rate much smaller than the optical transition rate.

The sample used in this work contained six QW structures, each of which consisted of an undoped $80 \AA$ thick CdTe QW separated by $200 \AA$ thick $\mathrm{Cd}_{0.7} \mathrm{Mg}_{0.3} \mathrm{Te}$ barriers from $500 \AA$ thick $\mathrm{CdTe} / \mathrm{Cd}_{0.7} \mathrm{Mg}_{0.3} \mathrm{Te}$ superlattices (SL) $(20 \AA / 20 \AA)$ (see Fig. 1a). Under the excitation above the superlattice miniband gap, different tunnelling probabilities for electrons and holes from the superlattice minibands into the QW via the $200 \AA$ thick barriers provide low-density excess electrons in the QWs, necessary for the formation of $X^{-}$.

The sample was mounted in an optical cryostat in the Faraday geometry with the magnetic field perpendicular to the QW plane. The $514 \mathrm{~nm}$ line of an argon-ion laser was used to generate the excitation above the superlattice miniband gap $\left(E_{\mathrm{g}}=1.8 \mathrm{eV}\right)$. For ODMR experiments the $70 \mathrm{GHz}$ microwa ves were chopped with $45 \mathrm{~Hz}$ and the synchronous changes in the luminescence intensity were recorded by a two-channel photon counter.

Figure $1 \mathrm{~b}$ shows the photoluminescence spectrum taken at the temperature $T=1.7 \mathrm{~K}$ and in the absence of magnetic field. There are two main lines observable: the one is identified as the heavy-hole exciton $X$ and the second line, $4.1 \mathrm{meV}$ lower in energy, is identified as $X^{-}$[5].

Figures $1 \mathrm{c}$ and $\mathrm{d}$ show ODMR spectra obtained by monitoring the microwave-induced changes of the $\sigma^{-}$and $\sigma^{+}$circularly polarized components of $X$ and $X^{-}$emissions, respectively. At the resonance magnetic field $\left(B_{\mathrm{res}}=3.424 \mathrm{~T}\right)$, the microwaves cause an increase in the $\sigma^{-}$emission intensity of $X^{-}$and simul-

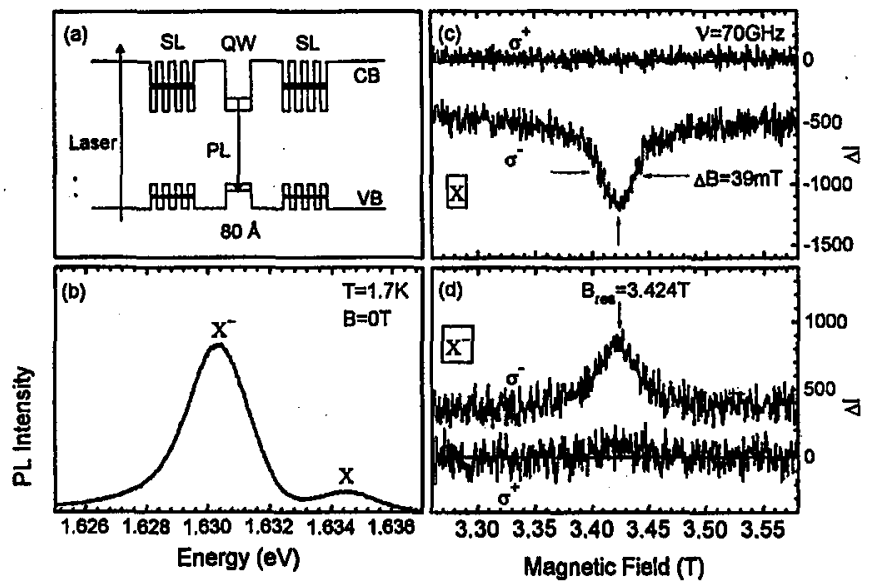

Fig. 1. (a) Scheme of the energy band structure for an $80 \AA \mathrm{CdTe} / 200 \AA \mathrm{Cd}_{0.7} \mathrm{Mg}_{0.3} \mathrm{Te}$ QW unit. (b) Photoluminescence intensity (in arbitrary units) taken at $T=1.7 \mathrm{~K}$ and $B=0 \mathrm{~T}$. (c), (d) ODMR signals (in arbitrary units) recorded by monitoring the microwave-induced changes in the $\sigma^{-}$and $\sigma^{+}$circularly polarized components of $X$ (c) and $X^{-}$(d) emission with microwa ve frequencies at $70 \mathrm{GHz}$. The ODMR lines with the linewidth of $\Delta B=39 \mathrm{mT}$ are locäted at $B_{\text {res }}=3.424$ T. From $\left|g^{*}\right| \mu_{\mathrm{B}} B=h \nu,\left|g^{*}\right|=$ $1.461 \pm 0.002$ is obtained. 
taneously a decrease in the $\sigma^{-}$emission intensity of $X$, whereas no changes are observed in the $\sigma^{+}$emission intensity of both $X$ and $X^{-}$. From $\left|g^{*}\right| \mu_{\mathrm{B}} B=h \nu$, we obtain $\left|g^{*}\right|=1.461 \pm 0.002$, which is in agreement with the value reported for conduction electrons in the $80 \AA \mathrm{CdTe} \mathrm{QW}[6]$. Based on the discussions below we assign the sharp ODMR lines to the EESR.

Two channels describing the formation and recombination of $X^{-}$(see Fig. 2) can be written as:

$$
\begin{aligned}
& \mathrm{e}_{|-1 / 2\rangle}+X_{|+1 / 2,-3 / 2\rangle} \rightarrow X_{|-3 / 2\rangle}^{-} \rightarrow \operatorname{photon}\left(\sigma^{-}\right)+\mathrm{e}_{|-1 / 2|}, \\
& \mathrm{e}_{|+1 / 2\rangle}+\mathrm{X}_{|-1 / 2,+3 / 2\rangle} \rightarrow X_{|+3 / 2\rangle}^{-} \rightarrow \operatorname{photon}\left(\sigma^{+}\right)+\mathrm{e}_{|+1 / 2\rangle} .
\end{aligned}
$$

In the presence of a magnetic field and low temperatures, the excess electrons populate mostly the $|+1 / 2\rangle$ states through thermalization during a long excess electron lifetime. In addition we observe that excitons have stronger $\sigma^{-}$ than $\sigma^{+}$emission intensities, indicating that excitons populate predominantly $|+1 / 2,-3 / 2\rangle=|-1\rangle$ states.

The recombination channel for $\sigma^{-}$polarization requires electrons in $|-1 / 2\rangle$ state, which has a small population. EESR causes an increase in the population of $|-1 / 2\rangle$ and, thus, it enhances the formation probability of the $|-3 / 2\rangle X^{-}$. Therefore, EESR is observed as an increase in the $\sigma^{-}$emission intensity of $X^{-}$and simultaneously a decrease in the $\sigma^{-}$emission intensity of $X$, because the enhanced emission of $X^{-}$occurs at an expense of $X$ emission intensity.

In the case of the recombination channel for $\sigma^{+}$polarization, $|+1 / 2\rangle$ electron state is strongly occupied and $|-1 / 2,+3 / 2\rangle=|+1\rangle$ exciton state has a low population. Therefore, the formation probability of the $\mid+3 / 2) X^{-}$is not very sensitive to changes in the population of $|+1 / 2\rangle$ electron state. This explains why the sharp electron ODMR line is not observed in $\sigma^{+}$polarization although EESR causes a decrease in the population of $|+1 / 2\rangle$ electron state.

The lifetime of direct excitons in $\mathrm{CdTe} /(\mathrm{Cd}, \mathrm{Mg}) \mathrm{Te} \mathrm{QWs}$ was measured to be $0.2 \mathrm{~ns}$ [7] and it was reported that the formation time and the lifetime of $X^{-}$

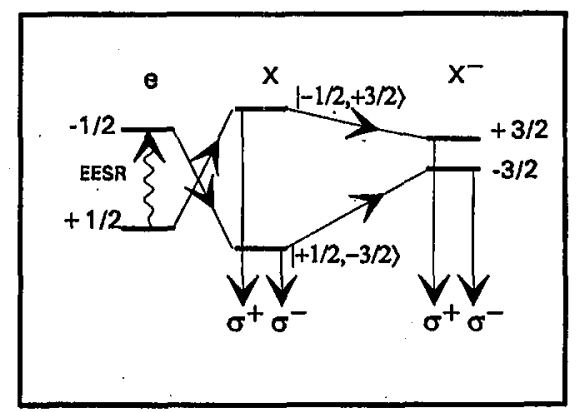

Fig. 2. Spin-dependent formation of $X^{-}$from excess electrons and excitons. The $g_{\mathrm{e}}^{*}$ value is taken negative and $g_{\mathrm{h}}^{*}$ much smaller and positive (see Ref. [6]). The excess-electron-spin resonance increases the $\sigma^{-}$emission intensity of $X^{-}$and simultaneously decreases the $\sigma^{-}$emission intensity of $X$. 
are both comparable to the exciton lifetime $[8,9]$. Usually microwave-induced spin transitions occur on sub-microsecond or even longer timescale. According to the usual prerequisite for ODMR, it should be impossible to detect ODMR on the fast direct $X$ or $X^{-}$emissions when the microwave-induced transition rate is much smaller than the optical decay rate. In fact, Eqs. (1) and (2) show that although the excess electron is involved in the formation of $X^{-}$, it is left with its spin conserved after the recombination of $X^{-}$. Microwaves can thus pump electrons from the lower spin state $(|+1 / 2\rangle)$ to the upper spin state $(|-1 / 2\rangle)$ continuously until the two spin states reach a new quasisteady population compared with that without microwave irradiation. A variation of the electron populations by EESR results in a change of the formation probability of $X^{-}$. Therefore, EESR can be detected via $X^{-}$or $X$ emission. From the above we see that the electron-spin dependent and the electron spin-conserving formation and recombination of $X^{-}$ make the optical detection of EESR feasible.

In conclusion, we have demonstrated that ODMR can be applied to study fast optical processes, such as the direct transitions of $X$ and $X^{-}$on the nanosecond timescale, where the microwave-induced transition rate is much smaller than the optical transition rate in type I QWs with an electron gas of low density. EESR can be observed with the detection on either the direct $X$ or $X^{-}$emissions. It can be expected that access to a new type of structures with small excess electron concentrations expands ODMR to be generally applied to investigate type I QWs with short-lived excitons.

We would like to express our gratitude to R. Kagerbauer and A. Gerand for processing the samples. This work is supported by the Deutsche Forschungsgemeinschaft via grant Os98-5 and by the grant from Volkswagen Foundation. One of us (C.Y. Hu) was additionally supported by the VW-Stiftung.

\section{References}

[1] For a review, see B.C. Cavenett, Adv. Phys. 30, 475 (1981).

[2] H.W. van Kesteren, E.C. Cosman, F.J.A.M. Greidanus, P. Dawson, K.J. Moore, C.T. Foxon, Phys. Rev. Lett. 61, 129 (1988).

[3] E. Glaser, J.M. Trombetta, T.A. Kennedy, S.M. Prokes, O.J. Glembocki, K.L. Wang, C.H. Chern, Phys. Rev. Lett. 65, 1247 (1990).

[4] B. Kowalski, P. Omling, B.K. Meyer, D.M. Hofmann, C. Wetzel, V. Härle, F. Scholz, P. Sobkowicz, Phys. Rev. B 49, R14786 (1994).

[5] D.R. Yakovlev, V.P. Kochereshko, R.A. Suris, H. Shenk, W. Ossau, A. Waag, G. Landwehr, P.C.M. Christianen, J.C. Maan, Phys. Rev. Lett. 79, 3974 (1997).

[6] Q.X. Zhao, M. Oestreich, N. Magnea, Appl. Phys. Lett. 69, 3704 (1996).

[7] W. Ossau, U. Zehnder, B. Kuhnheinrich, A. Waag, T. Litz, G. Landwehr, R. Hellman, E.O. Göbel, Superlattices Microstruct. 16, 5 (1994).

[8] K. Kheng, R.T. Cox, V.P. Kochereshko, K. Saminadayar, S. Tatarenko, F. Bassani, A. Franciosi, Superlattices Microstruct. 15, 253 (1994).

[9] Arza Ron, H.W. Yoon, M.D. Sturge, A. Manassen, E. Cohen, L.N. Pfeiffer, Solid State Commun. 97, 741 (1996). 